\title{
Application of Nano Antibacterial Materials in the Passenger Seat of the City Bus
}

\author{
Xiaojing Yang, Kunqian Wang \\ Faculty of Art and Communication, \\ Kunming University of Science And Technology, \\ Yunnan, China
}

\begin{abstract}
The danger to public health and safety caused by the timely sterilization failure of harmful bacteria on the seat of the city bus due to frequent movement of the passengers is explored in this paper. According to different types and different materials of the passenger seats, combined with the advantages and disadvantages between inorganic nano silver antimicrobial agent and photocatalytic antibacterial $\mathrm{TiO}_{2}$ antibacterial agent, it is believed that the modified nano $\mathrm{TiO}_{2}$ antibacterial agent is more suitable for being added into the antibacterial plastics and the combining with nanoantibacterial fiber, and which has broad application prospects on the bus seat materials.
\end{abstract}

Keywords-nanotechnology; antibacterial materials; city bus; passenger seat

\section{INTRODUCTION}

Recently, the Ebola virus has been rampant in Africa. The bacterial variation and the spread of the disease caused great panic among people, in view of the largescale outbreak of SARS, avian influenza and H7N9 virus, and other infectious diseases, people have higher and higher requirements for the public health, especially travel safety and hygiene.

City bus is an important symbol of modern urban civilization; the sanitary conditions on the bus cannot solve the real problems if only through simple hygiene disinfection and cleaning process. The passenger seat is one of the most frequently used facilities in the bus, and the corner is easy to be forgot when the bus is cleaned. In 2010, several strains of the mushroom grew on the two side of the passenger seat on Wuhan Bus 755, which was caused by the halfway cleaning and the wanton growth of microorganisms. For the sake of the passengers' safety and health, the use of antibacterial nanotechnology has been extremely urgent in solving the problem of the bacteria left on the seat.

\section{ANTIBACTERIAL NANOMATERIALS}

Nanotechnology is the high science and technology at the end of the 1980s, and it has a rapid development in recent years in various fields. The core component of antibacterial nanomaterials is antibacterial agent. On the city bus, the frequent movement of people causes all sorts of bacteria left on the passenger's seat. The passenger seat made from the antibacterial nanomaterials enables the seat with inherent antibacterial properties, therefore the seat has the effect of inhibiting bacterial reproduction or destruction of bacteria cell, effectively avoiding the spread of bacteria and other microorganisms, and preventing cross infection of the bacteria between passenger and the seat, the seat and a passenger, and enhancing mechanical properties of antibacterial seat materials.

Nano-antibacterial agent has many types, which can be classified into natural biological antibacterial agent, organic chemistry antibacterial agent, inorganic antibacterial agent, and composite polymer antibacterial agent. At present, the natural antimicrobial agent generally refers to chitosan and its derivatives, due to its poor heat resistance, it needs further research and development of the researchers; organic antibacterial agent is mainly the organic nitrogen ion compound, which is the main germicidal mechanism, destroying the cell membrane, the protein denaturation, and metabolic block. In spite of many types of organic antibacterial agent, most of them have the disadvantages of volatility and toxicity, poor heat resistance, and easy solution [1].

The inorganic antimicrobial agent is prepared by the combination of antibacterial performance of silver, titanium, and other metals and carrier through certain processing technology and technical means. And it can be divided into the following two types according to its antibacterial mechanism: one is the antimicrobial agent dominated by metallic silver ion, main mechanism dominated by contact sterilization, and the chemical equation is shown in Figure 1. The other is the antimicrobial agent dominated by $\mathrm{TiO}_{2}$ photocatalytic titanium. Inorganic antibacterial agent has low toxicity, good broad-spectrum antimicrobial resistance, good heat resistance, etc., and it is widely used in domestic products.

\section{APPLICATION OF ANTIBACTERIAL AGENT IN COMBINATION WITH DIFFERENT MATERIALS}

Antibacterial materials are new materials prepared by quantitative antimicrobial agent in combination with materials and have highly effective inhibition for the microorganism in the daily use, and they prevent crossinfection of disease, and kill bacteria left on the materials.

Materials of the most passenger seat are hard plastic, a small parts use the cushion and cushion in the seat back and soft fiber products (as shown in Figure 2). According to different types and different materials of the passenger seat, the existing mature nanomaterials are analyzed, applied into the passenger seat of the city bus. 
$\mathrm{SH}$

Enzym

$$
+
$$

$\mathrm{SH}$
SAg

Enzyme

SAg

Figure 1. Silver ion sterilization schematics.

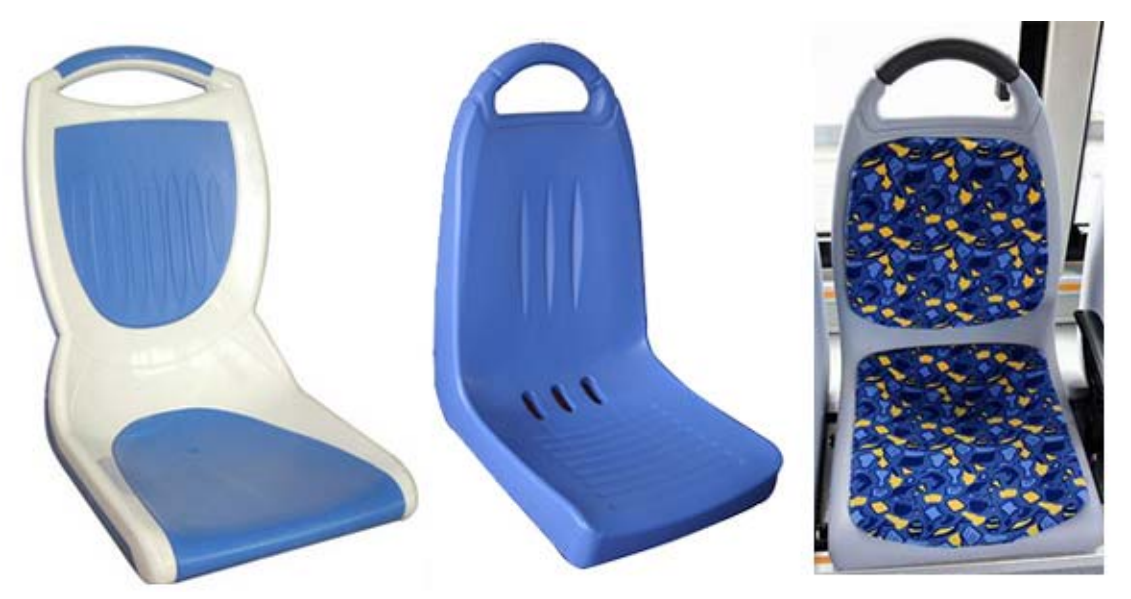

Figure 2. Passenger seat of the city bus.

\section{A. Antibacterial Plastic}

Nano-antibacterial plastic is a new material, which itself has inherent antibacterial, antimicrobial, bactericidal function, can be widely applied in many fields of industrial manufacturing, daily life, and therefore, it has a solid scientific foundation in the antimicrobial plastic seat.

Plastic antibacterial agent refers to adding quantitative antibacterial agent or plastic antibacterial master batch and other new plastic additives which enable the plastic to have a long-term antibacterial and germicidal effect. Currently, inorganic antibacterial agent can adapt to high temperature required in the plastic molding. After the

$$
\begin{array}{ll}
\text { The quantitive antibacterial } \\
\text { agent is added to the material } \\
\text { and mixed. }
\end{array}
$$

pilot study of the researchers, the conventional antibacterial metallic copper sulfate, zinc nitrate, and the like powders are difficult in combination with thermoplastics, and the antibacterial plastic master batch obtained by an inorganic nano-antibacterial agent powder by special treatment can applied into the plastics, and has a good compatibility, and it is conducive to the uniform distribution of antibacterial agent in the material [2, 3]. Its process can be simply shown in Figure 3.

Directly mixed (plastic products)

Master batch production (fiber, plastics)

Figure 3. Processing schematic of plastic antibacterial agent.

Inorganic antibacterial agent consists of metal ion: $\mathrm{Ag}$ $+, \mathrm{Cu}^{2+}, \mathrm{Zn}^{2+}$, and Photocatalysis: such as $\mathrm{TiO}_{2}$ and $\mathrm{ZnO}$, $\mathrm{ZrO}^{2}$. In the metal ion antibacterial agent, Ag ion has strong heat resistance, has high antibacterial effect on most deadly bacteria, it is known as the most used antibacterial agent in the research. But metallic silver is easy to be oxidized in the air and changes color, so it has the characteristic of poor light stability, the high dosage, and the higher cost. And the release of the heavy silver ion will be harmful to the environment and human health [47].

In antibacterial agent of photocatalytic types, currently, the most widely studied photocatalytic antibacterial agent is $\mathrm{TiO}_{2}$, and it has no stimulation to the skin, and long antibacterial effect and broad spectrum of antibacterial properties. The mechanism of photocatalytic antibacterial agent can be simply shown as follows:

$$
\begin{aligned}
& \mathrm{TiO}_{2} / \mathrm{ZnO}+\mathrm{h} \mathrm{v} \rightarrow \mathrm{e}^{-}+\mathrm{h}^{+} \\
& \mathrm{h}^{+}+\mathrm{H}_{2} \mathrm{O} \rightarrow \cdot \mathrm{OH}+\mathrm{H}^{+} \\
& \mathrm{e}^{-}+\mathrm{O}_{2} \rightarrow \cdot \mathrm{O}_{2}^{-}
\end{aligned}
$$

Because ordinary $\mathrm{TiO}_{2}$ antibacterial agent has antibacterial function only under ultraviolet light, so it has very important significance to study the modification of nano composite $\mathrm{TiO}_{2}$ antibacterial agent and its application in antibacterial plastic. Deng Yuming and other researchers developed $\mathrm{TiO}_{2}$ nano-antibacterial agent, and achieved a 
major breakthrough; trace amounts of metal or nonmetal added into the $\mathrm{TiO}_{2}$ antibacterial agent can significantly improve the photocatalytic activity and sterilization ability of the $\mathrm{TiO}_{2}$ in the range of visible light. The modified antibacterial agent has good compatibility with PE, PP, and other polymer materials, and can be applied in the plastic material of nano-antibacterial passenger seat of the city bus.

Most of the passenger seats in the city bus select plastic materials, which components are organic macromolecules. Seat will produce many scratches after people's long time friction, which is easy to make the bacteria growing on it. Therefore the antibacterial agent selected not only needs to have a long-term effective antibacterial property, and have on very strong sterilization ability for Escherichia coli, Staphylococcus aureus, salmonella, mould, and other germs; it should maintain the cleanness of material itself; non-toxicity, odorless, harmless to the human body and it should not be decomposed in the process of plastic processing, never degenerating and low production cost.



Figure 4. Processing flow chart of plastic antibacterial agent.

\section{B. Antibacterial Fiber}

Because of difference in social environment, cultural background, traffic and road conditions of each country, the design of modern buses will be specifically based on the actual situations of the city. Such as in Edinburgh, the bus seats use grid fabric sofa. In China, part of the bus seats is equipped with cushion and seat back cushion to increase the comfort for passengers. Fabric seat is not easy to clean, microorganism is easy to breed, therefore, the introduction of nanofibers makes the fabric itself have the ability to inhibit bacteria, microbial, improve health conditions inside the bus, providing certain security for passengers.

Antibacterial fiber refers to the use of textile fiber porous physical structure and chemical structure of the polymer, by physical or chemical methods. Applicable antibacterial agent is inserted in the textile fiber, so that the fiber textiles have the function of killing bacteria or inhibiting the growth of bacteria breeding, keeping efficient and durable antibacterial deodorization effect. Currently, China can batch produce two kinds of antibacterial fibers: one is the inorganic antibacterial fiber represented by the synthetic fiber containing silver zeolite antibacterial materials. The other is the series antibacterial fiber co-developed by the organic antibacterial agent [810].

The preparation of antibacterial fiber has three types in general: melt spinning method, composite spinning method, and chemical modification spinning method. These three preparation processes can be simply shown in Figure 4.

The ideal antibacterial materials not only have a broad spectrum for harmful microorganisms and the highly effective suppression function, can remove bad smell, destroy microbial cell structure; it should also have the requirement of the following three aspects: first, security: the product is produced for people's use, antibacterial materials must be non-toxic and harmless to people and environment; Second, compatibility: it shall not affect the performance, appearance of the finished products, and it should not have the ability to enhance product performance; Third, durability: the product should meet longterm antimicrobial requirements of resistance to friction and consumption in the process.

\section{OUTLOOK AND PROSPECTS}

City bus is one of the public transport facilities with social welfare nature and with characteristic of groups. The introduction of the passenger seat containing nanoantibacterial technology in the public transport system has improved the self-cleaning disinfection of the seat, reduced the growth of bacteria on the seat, and the secondary spread of pollution, which will greatly reduce the amount of labor of the bus cleaning staff and cleaning agents and water resource consumption, reduce seat wear and update, achieving green energy-saving and environmental protection, and the protection of public health. The research and preparation of new antimicrobial plastic materials, the development and application of the antibacterial mechanism of the antimicrobial fiber, are one of the future directions of nano-antibacterial seats research.

With advance of urbanization, low-carbon living, green travel has become an inevitable trend of modern urban life, the application of nano-antibacterial materials in the passenger seat of city bus will attract more residents to take the bus. Sterilization, bacteriostasis of nanomaterials will provide the 
majority of passengers with health protection and will have a good social benefit. The wide application of nanoantibacterial materials in the automotive industry will bring good economic benefits.

\section{REFERENCES}

[1] Xia Jinlan, Wang Chun, Liu Xinxing, Research on antimicrobial agents and their mechanisms of actions. Journal of Central South University, 35(1), 2004

[2] Pan Xiaoyong, Du Yanyan, Chen Wei, et al., Applied properties of $\mathrm{Ag} / \mathrm{TiO}_{2}$ in Antibacterial plastics. Engineering Plastics Application, 11(40), pp. 101-102, 2012.

[3] S.H. Jeong, Y.H. Hwang, S.C. Yi, Antibacterial properties of padded $\mathrm{PP} / \mathrm{PE}$ nonwovens incorporating nanosized silver colloids. Journal of Materials Science, 40(5), pp. 5413-5418, 2005.
[4] Chun Hu, Jian Guo, Jiuhui Qu, et.al., Photocatalytic degradation of pathogenic bacteria with AgI/TiO2 under visible light irradiation, Langmuir, 23(9), pp. 4982-4987, 2007.

[5] Radhesh Kumar, Helmut Munstedt, Silver ion release from antimicrobial polyamide/silver composites, Biomaterials, 26, pp. 20812088, 2004.

[6] E. Manzini, F. Jegou, Sustainable Everyday: Scenarios of Urban Life. Edizioni Ambiente, Milano, 2003.

[7] A. Fujishima, K. Honda, Electrochemical photolysis of water at a semiconductor electrode. Nature, 238(5358), pp. 37-38, 1972.

[8] H.S. Jia, et a1., The structures and antibacterial properties of nano-SiO2 supposed silver/zinc-silver materials. Dental Materials, 24, pp. 244 249, 2008.

[9] Liu Weishi, Development of antibacterial fiber and application of antibacterial textiles. Chemical Fiber\&Textile Technology, 9(3), 40, 2011.

[10] Chen Daojiang, Ma Zhengsheng, The development of antibacterial fiber and textiles, Guangxi Textile Science Technology, 35(1), 2006. 